there were 11 Grade 2 pressure ulcers from 109 admissions; in period 2 there were 5 pressure ulcers from 105 admissions. Benefits of using total face masks for NIV delivery were also noted with those patients who were poorly complaint with the standard NIV full face mask to prevent treatment failure.

Conclusions An early prophylactic pressure-relieving dressing and a reactive change to a pressure-avoidance mask for identified Grade 1 pressure sore, can reduce the chance of developing Grade 2 pressure ulcers for patients using NIV acutely. Further studies including longitudinal data on a proactive prevention approach adjusted for acute NIV duration for NIV-related nasal bridge pressure ulceration are needed to confirm the utility of this approach.

\section{P74 NON-INVASIVE VENTILATION DELIVERED ON A STANDARD RESPIRATORY UNIT COMPARED TO USE IN LEVEL 2 CARE SETTING: IS THERE AN IDEAL SERVICE DELIVERY MODEL?}

${ }^{1}$ A Jayadev, ${ }^{2} \mathrm{~K}$ Mcvinnie, ${ }^{2}$ M Moonsie. ${ }^{1}$ The Royal Free Hospital, London, UK; ${ }^{2}$ North Middlesex University Hospital, London, UK

\subsection{6/thoraxjnl-2016-209333.217}

Introduction BTS guidelines recommend Non-Invasive Ventilation (NIV) should take place in a clinical environment with enhanced monitoring, predicting $20 \%$ of all cases may need level 2 or 3 care. $^{1}$ However, current practice varies between and within NHS organisations. A management led service change within our Trust in 2013 enabled us to test the null hypothesis that there is no significant difference in mortality of COPD patients requiring NIV on an open respiratory-led unit (level 1 care), compared to a closed, anaesthetist led Level 2 setting (PCU, Progressive Care Unit)

Methods An electronic search was performed to find patients on PCU whom received NIV between 1st January and 30th November 2014. Inclusion criteria were patients that had received NIV for COPD exacerbations solely. Data from the physician led respiratory unit between Jan-Nov 2011 was prospectively collected, and the two datasets compared.

Results In the respiratory unit 75 patients were admitted for NIV of which 54 met the criteria for inclusion in the analysis. In the PCU group 110 patients were admitted between Jan-Nov 2014. of which 55 were included for analysis.

Samples were matched in gender, with no significant difference between groups. The average age of patients treated in PCU was 69.8 years, and 74.4 years on the respiratory unit, which is statistically significant (Mann-Whitney U Test, $\mathrm{p}=0.012$ ). The mortality on PCU was $27.2 \%$ compared to $20.4 \%$ on the respiratory unit, which was not statistically significant. Mean $\mathrm{pH}$ on PCU was 7.33 compared to 7.24 on the respiratory Unit, which is statistically significant. Mean $\mathrm{pCO}_{2}$ was 10.06 on $\mathrm{PCU}$, and 10.5 on the respiratory unit, which is statistically significant. Average length of stay of ward patients was 15 days, compared to 11.4 days on PCU, which was not statistically significant.

Conclusions NIV delivered on a physician-led respiratory unit was not inferior in mortality and length of stay compared with a closed, ITU-led service. Interestingly we found a significant difference in age of patients being treated with NIV, with significantly older patients receiving this on ward with no difference in overall mortality.

\section{REFERENCE}

1 BTS Guidelines Management of AHRF 2016. http://www.brit-thoracic.org.uk

\section{P75 PATIENT EXPERIENCE OF NON-INVASIVE VENTILATION: A QUALITATIVE STUDY}

N Goldman, L Richardson, S Blakey, L Staveacre, SAA Bloch. Imperial College Healthcare NHS Trust, London, UK

\subsection{6/thoraxjnl-2016-209333.218}

Introduction Non-invasive ventilation (NIV) is an effective treatment for acute type 2 respiratory failure, often avoiding intubation and improving mortality. However many patients struggle to tolerate NIV. There is limited understanding of patients' or their relatives/carers subjective experience of NIV. As good patient experience is increasingly recognised to reflect high-quality care we conducted an in-depth experience-based questionnaire aiming to identify key concerns of patients, and their relatives/carers, treated with NIV, which would reflect potential targets for service improvement.

Method In a qualitative, exploratory study patients started acutely on NIV were identified. Patients and relatives/carers completed a questionnaire with both free text and Likert style responses. Data were analysed using thematic analysis.

Results 20 questionnaires were completed (15 patients, 5 relatives). From the responses we identified key themes. Emotional responses were positive and negative. Positively - all patients and carers felt that NIV had helped. However whilst all carers would wish their relatives to have NIV again, 2 of the patients felt they would not. Negative emotional responses were related to fear and anxiety of NIV. A significant theme emerged surrounding the physical discomfort of NIV. Descriptions of NIV are represented in Figure 1. Patients and relatives identified that negative feelings were partly due to lack of understanding. Only 9 patients felt that they were involved in decision making and only 6 felt that NIV had been adequately explained. 11 patients and all relatives felt that written information would be beneficial. Finally a further theme described different levels of competence between staff and across wards and the varying degrees of feeling safe that this created.

Conclusion This study enabled us to identify key areas to address when considering quality improvement for NIV service delivery. Whilst our sample size is small, and biassed towards survivors, the themes are strong and add significantly to the available literature. Some aspects of NIV are non-modifiable however focus on patient involvement and experience to provide high-quality care may facilitate improved experience and outcomes. We aim to address these points by expanding on this work in an experiencebased co-designed project, funded by CLAHRC. 


\section{Correction}

Jayadev A, Mcvinnie K, Moonsie I. P74 Non-invasive ventilation delivered on a standard respiratory unit compared to use in level 2 care setting: is there an ideal service delivery model? Thorax 2016;71:A125. doi:10.1136/thoraxjnl-2016-209333.217

Corrections have been made to the 'Methods', 'Results' and 'Conclusions' section of this abstract. The changes are in bold.

Methods

An electronic search was performed to find patients on PCU whom received NIV between 1st January and 30th November 2014. Inclusion criteria were patients that had received NIV for COPD exacerbations solely. Data from the physician led respiratory unit between Jan-Nov 2011 was prospectively collected, and the two datasets compared.

Results

In the respiratory unit 75 patients were admitted for NIV of which 39 met the criteria for inclusion in the analysis. In the PCU group 110 patients were admitted between Jan-Nov 2014 of which 55 were included for analysis. Samples were matched in gender, with no significant difference between groups. The average age of patients treated in PCU was 69.8 years, and 69 years on the respiratory unit (Mann-Whitney $U$ Test, $p=0.012$ ). The in-hospital mortality on PCU was $27.2 \%$ compared to $15 \%$ on the respiratory unit $(p=0.15)$. Mean $p H$ on PCU was 7.31 and 7.24 on the respiratory unit $(p=0.186$, not significant). Mean pCO2 was lower at $9.16 \mathrm{kPa}$ on PCU compared to $10.7(\mathrm{p}=0.0703)$ on the respiratory unit, which is not statistically significant. Average length of stay was equal in both units at 12 days.

Conclusions

NIV delivered on a physician-led respiratory unit was not inferior in mortality and length of stay compared with a closed, ITU-led service in our hospital. Interestingly, the ward patients were sicker, with a lower mean $\mathrm{pH}$ and higher mean $\mathrm{pCO} 2$ but had a lower mortality, although not statistically significant. This supports a model where acute COPD patients needing NIV can be managed safely in a respiratory ward area with enhanced staffing as outlined in BTS guidelines. This could have continuity of care and cost benefits. 